\title{
Role of Magnetic Fields in Star Formation
}

\author{
Richard M. Crutcher \\ Department of Astronomy, University of Illinois, Urbana, IL 61801, USA \\ email: crutcher@illinois.edu
}

\begin{abstract}
I describe two recent projects to test star formation theory using Zeeman observations. First, using Bayesian analysis, the probability distribution function of the magnitude of the total magnetic field strength $B_{t}$ and its dependence on volume density $n(H)$ were inferred from Zeeman observations of the line-of-sight strengths $B_{z}$. The result was that from one molecular cloud to another $B_{t}$ ranges uniformly between values close to zero and a maximum $B_{0}$, and that $B_{0}$ scales as $n^{2 / 3}$. Second, observations of the ratio of the mass/flux $(M / \Phi)$ between the core and envelope regions of four dark clouds yielded values $<1$. All of these results disagree with predictions of the strong magnetic field, ambipolar diffusion driven theory of star formation.
\end{abstract}

Keywords. ISM: magnetic fields, stars: formation, techniques: polarimetric

\section{Introduction}

The role of magnetic fields in star formation remains unclear. One reason is that radio Zeeman observations yield only the line-of-sight component $B_{z}$ of the magnetic vector $\mathbf{B}$. Here we very briefly describe two efforts to overcome this limitation in order to test star formation theory with observations of magnetic field strengths.

\section{Bayesian analysis of Zeeman data}

It is possible to infer statistical information about the total magnetic field strength $B_{t}$ in a sample of interstellar clouds by making assumptions. One assumption is that the direction of $\mathbf{B}$ is random from cloud to cloud, so that the set of possible observed $B_{z}$ range from zero up to the full magnitude $B_{t}$ of $\mathbf{B}$. Another assumption concerns the probability distribution function (pdf) of the magnitude of the total strength of the 3D magnetic field and its relation to the pdf of the observed $B_{z}$. Heiles \& Crutcher (2005) have discussed this assumption. They considered four analytic functions to describe the pdf of $B_{t}$ : a Kronecker delta function, a flat or uniform distribution, a weighted Gaussian function, and a Gaussian function. All assumed possible forms for the pdf yield mean and median values for $B_{z}$ roughly equal to $0.5 B_{t}$, so if one is only interested in inferring the approximate mean or median of $B_{t}$ from a set of $B_{z}$ measurements, the form of the distribution of $B_{t}$ within the set of clouds does not matter very much.

However, having only approximate information about the mean or median value of $B_{t}$ significantly limits our knowledge of interstellar magnetic fields. In order to overcome this limitation, Crutcher et al. (2010) carried out a Bayesian statistical analysis of H I, OH, and CN Zeeman surveys of diffuse and molecular clouds. Although a number of studies were carried out, here I give the results of only the most comprehensive study. The relationship between the cloud density $n$ and the maximum magnetic field in a cloud was taken to be $B_{\max }(n)=B_{0}$ for $n<n_{0}, B_{\max }(n)=B_{0}\left(n / n_{0}\right)^{\alpha}$ for $n>n_{0}$. Finally, the pdf of $B_{t}$ was parameterized by a flat pdf with the smallest values cut off depending on the value of a parameter $f$; then $f B_{0}<B_{t}<B_{0}$. The flat pdf over the full range 0 to $B_{0}$ is given by $f=0$ and the delta-function pdf by $f=1$, so this parameterization covers a wide range in possible pdf's. Results for the median values of the four parameters were: $B_{0} \approx 10 \mu \mathrm{G}, n_{0} \approx 300 \mathrm{~cm}^{-3}, \alpha \approx 0.67$, and $f \approx 0.03$ (which is essentially the flat pdf over the full range $0<B_{t}<B_{0}$. 


\section{Core/envelope mass-to-flux ratio measurements}

Crutcher et al. (Crutcher, Hakobian \& Troland (2009)) carried out OH Zeeman observations toward the envelope regions surrounding four molecular cloud cores for which detections of $B_{z}$ had been achieved in the same lines, and evaluated the ratio of mass to magnetic flux $(M / \Phi)$ between the cloud core and envelope. This relative $M / \Phi$ measurement reduces uncertainties in previous studies, such as the angle $\theta$ between $\mathbf{B}$ and the line of sight. They calculated $\mathcal{R} \equiv[M / \Phi]_{\text {core }} /[M / \Phi]_{\text {env }}=\left[N_{O H} /\left(B_{z} \cos \theta\right)\right]_{\text {core }} /\left[N_{O H} /\left(B_{z} \cos \theta\right)\right]_{\text {env }}$. With the assumption that $\theta_{\text {core }} \approx \theta_{\text {env }}$, as predicted by idealized strong-field models, the unknown $\theta$ s drop out, so $\mathcal{R}$ can be directly found from observations. The result was that for all four clouds, $\mathcal{R}<1$. The idealized ambipolar diffusion theory of core formation requires the ratio of the central to envelope $M / \Phi$ to be approximately equal to the inverse of the original subcritical $M / \Phi$, or $\mathcal{R}>1$. The probability that all four of these clouds have $\mathcal{R}>1$ is $3 \times 10^{-7}$; the results are therefore significantly in contradiction with the hypothesis that these four cores were formed by ambipolar diffusion.

Mouschovias and Tassis (unpublished) strongly criticized this result, first arguing that the apparent variation of $B_{z}$ among the four positions observed for each envelope proved that the $\theta$ s varied greatly due to large-scale bending of field lines. Hence, ignoring the putative variation in the measured $B_{z}$ led to too small an uncertainty in $\mathcal{R}$. However, the observed variation in the $B_{z}$ was at the $2-3 \sigma$ level - that is, statistically insignificant. Second, they carried out their own analysis of the data incorporating the putative variations in $\left(B_{z}\right)_{e n v}$, which led to larger uncertainties in the $\mathcal{R}$ such that $\mathcal{R}>1$ was not excluded. However, they employed the relationship above for $\mathcal{R}$ that assumes that $\theta_{\text {core }} \approx \theta_{\text {env }}$ so the $\cos \theta$ s divide out. Use of the expression for $\mathcal{R}$ without the individual $\theta$ s while assuming that the $\theta$ s vary widely from position to position is internally inconsistent. For both reasons, their criticism is therefore invalid.

\section{Conclusions}

The Bayesian analysis found that the distribution of the $B_{t}$ ranges from very small values up to a $B_{\max }$ that scales with density approximately as $n^{2 / 3}$. The power-law scaling is not consistent with a strong magnetic field model, but rather with one in which magnetic energy is much weaker than gravity (highly supercritical). Also, the fact that a significant population of molecular clouds must have very small magnetic field strengths implies that for many molecular clouds, magnetic fields do not dominate. The relative $M / \Phi$ experimental results were not consistent with the "idealized" strong field, ambipolar diffusion theory of star formation. The conclusion is that the role of magnetic fields in star formation is complicated and diverse, with much work remaining to be done.

\section{References}

Crutcher, R. M., Wandelt, B., Heiles, C., Falgarone, E., \& Troland, T. H. 2010, ApJ submitted Crutcher, R. M., Hakobian, N., \& Troland, T. H. 2009, ApJ 692, 844

Heiles, C. \& Crutcher, R. M. 2005, Cosmic Magnetic Fields, Lecture Notes in Physics (Heidelberg: Springer), vol. 218, p. 23 